\title{
Effectiveness and safety of ipilimumab therapy in advanced melanoma: evidence from clinical practice sites in the US
}

\author{
Kim A Margolin, MD, ${ }^{a}$ Ahmad Tarhini, MD, PhD, ${ }^{b}$ Sumati Rao, $\mathrm{PhD},{ }^{\mathrm{c}}$ Monica Katyal, \\ JD, MPH, ${ }^{\mathrm{d}}$ I-Fen Chang, PharmD, ${ }^{\mathrm{c}}$ Douglas B Johnson, MD, ${ }^{\mathrm{e}}$ Sekwon Jang, MD, ${ }^{\mathrm{f}}$ Joseph I \\ Clark, MD, ${ }^{\mathrm{g}}$ and David McDermott, $\mathrm{MD}^{\mathrm{h}}$
}

${ }^{a}$ University of Washington, Seattle, Washington; ${ }^{b}$ University of Pittsburgh, Pittsburgh, Pennsylvania; 'Bristol-Myers Squibb, Plainsboro, New Jersey; ${ }^{\mathrm{d}}$ Medical Data Analytics, Parsippany, New Jersey; ${ }^{\mathrm{e}}$ Vanderbilt University Medical Center, Nashville, Tennessee; ${ }^{\mathrm{f}}$ Washington Cancer Institute at MedStar Washington Hospital Center, Washington, DC; ${ }^{\mathrm{g}}$ Loyola University Medical Center, Cardinal Bernardin Cancer Center, Maywood, Illinois; and heth Israel Deaconess Medical Center, Boston, Massachusetts

Background Ipilimumab was approved in 2011 by the US Food and Drug Administration in 2011 for the treatment of unresectable or metastatic (advanced) melanoma, although pivotal data using the approved $3 \mathrm{mg} / \mathrm{kg}$ monotherapy q3w $\times 4$ were available only for patients with previously treated disease.

Objective To investigate patient and disease characteristics, survival outcomes, and safety in treatment-naïve patients receiving ipilimumab therapy.

Methods Adult patients with treatment-naïve advanced melanoma who received $\geq 1$ dose of ipilimumab $3 \mathrm{mg} / \mathrm{kg} \mathrm{during} \mathrm{April}$ 2011 -Sept 2012, with $\geq 12$ months having elapsed since the start of treatment, were identified from 34 US sites. Personnel from each study site retrospectively abstracted existing data from individual patient medical records, which were collected and validated by an independent research organization.

Results In all, 273 patients were included in the study. The median age of the total study population was 64 years (range, 26-91), and $64.8 \%$ were men. At diagnosis, $56.0 \%$ were stage IV Mlc, and $12.1 \%$ had brain metastases. 50 patients had a BRAF mutation, 181 were BRAF wild-type, and BRAF status was not known for $42.78 \%$ of patients received all 4 planned doses of ipilimumab. Median survival from initiation of ipilimumab treatment was 14.5 months (95\% confidence index [Cl], 12.9-18.7). The overall one-year survival rate was $59.2 \%(95 \% \mathrm{Cl}, 53.0-64.8)$; and $71.0 \%$ and $54.9 \%$ for patients with BRAF-mutated and wild-type tumors, respectively. Adverse events of any grade, grade 3, and grade 4 occurred in 164 patients (60.1\%), 45 (16.5\%), and 8 $(2.9 \%)$, respectively. The most common grade 3 or 4 adverse events were colitis $(4.0 \%)$, fatigue (2.9\%), and diarrhea (1.5\%). Drugrelated adverse events were primarily immune-related and occurred in 147 patients $(53.8 \%)$, including grade $3 / 4$ in $15.7 \%$ of patients $(13.9 \%$ and $1.8 \%$, respectively). No deaths were attributed to ipilimumab.

Conclusions This observational study provides real-world, clinical practice evidence supporting improved survival with the approved ipilimumab $3 \mathrm{mg} / \mathrm{kg}$ monotherapy in patients with treatment-naïve advanced melanoma, including prolonged survival in some patients. The safety profile was consistent with that reported in clinical trials.

Funding/sponsorship Bristol-Myers Squibb. Dr Johnson is supported by NIH training grant K12 CA 0906525.

\section{$\mathrm{T}$} he incidence of melanoma, the most serious form of skin cancer, is rising globally. ${ }^{1,2}$ Although most patients are diagnosed and treated at an early stage of the disease and have a good prognosis, survival among patients with unresectable or metastatic (advanced) melanoma remains poor. In the United States, an estimated 9,940 deaths from melanoma will occur in $2015 ;^{1}$ in Europe, melanoma accounts for an estimated 20,000 deaths annually. ${ }^{2}$
Patients with advanced melanoma historically had a dismal prognosis, with median survival of less than a year. ${ }^{3,4}$ In a meta-analysis of 2,100 patients with advanced melanoma from 42 phase 2 cooperative group clinical trials with accrual closing between 1975 and 2005, the median overall survival (OS) was 6.2 months (95\% confidence interval [CI], 5.96.5), with $25.5 \%$ (95\% CI, 23.6-27.4) alive at 1 year. $^{3}$ Another recent systematic review estimated median OS for stage IV melanoma of about 8 months,

Accepted for publication March 13, 2015. Correspondence: Sumati Rao, PhD; sumati.rao@bms.com. Disclosures: Drs Rao and Chang are employees of Bristol-Myers Squibb. Dr Clark receives grants and speakers bureau fees from Bristol-Myers Squibb. Dr McDermott reports compensation from Bristol-Myers Squibb for advisory board attendance. Dr Tarhini reports grants received and compensation from Bristol-Myers Squibb for advisory board attendance. Dr Margolin and Ms Katyal receive grants from Bristol-Myers Squibb. Dr Jang and Dr Johnson report nothing to disclose. JCSO 2015;13:131-138. C2015 Frontline Medical Communications. DOI 10.12788/jcso.0124. 
with about $10 \%$ of patients surviving more than 5 years. ${ }^{4}$ Beginning in 2010, however, phase 3 studies of several novel agents showed statistically improved OS in patients with advanced melanoma. ${ }^{5-7}$ These findings further validated the use of immunotherapy and introduced molecularly targeted therapy for the treatment of advanced melanoma, resulting in the availability of several new agents for use in routine clinical practice. ${ }^{8}$

The first of these new agents was the first-in-class immune checkpoint inhibitor ipilimumab, a human monoclonal antibody. Ipilimumab targets the cytotoxic T-lymphocyte-associated antigen 4 (CTLA-4) receptor, blocking its inhibitory mechanisms and thereby augmenting T-lymphocyte-mediated antitumor immune responses. Ipilimumab monotherapy, $3 \mathrm{mg} / \mathrm{kg}$ every 3 weeks for 4 doses, was approved in 2011 by the US Food and Drug Administration on the basis of significant improvements in OS demonstrated in 2 pivotal, phase 3 randomized, double-blind trials in patients with advanced melanoma. The first study was conducted in previously treated patients; ipilimumab $3 \mathrm{mg} / \mathrm{kg}$ prolonged OS, whether administered as monotherapy (median, 10.1 months) or combined with a glycoprotein 100 (gp100) peptide vaccine (median, 10.0 months), over gp100 alone (median, 6.4 months; hazard ratio $[\mathrm{HR}]$ for death for ipilimumab alone vs gp100 alone, $0.66 ; \mathrm{P}=.003$ ). ${ }^{5}$ Furthermore, there were $20.3 \%$ (45.6 vs $25.3)$ and $9.8 \%$ (23.5 vs 13.7$)$ more patients alive in the ipilimumab-only arm than in the gp100 arm at 1 year and 2 years, respectively. In the second trial, patients with treatment-naïve disease who received a concurrent regimen of ipilimumab $10 \mathrm{mg} / \mathrm{kg}$ and dacarbazine had improved OS over single-agent dacarbazine (median, 11.2 vs 9.1 months; $\mathrm{HR}, 0.72 ; \mathrm{P}<.001) .{ }^{6}$ Higher survival rates were also seen in this trial in the patients treated with ipilimumab-dacarbazine compared with dacarbazine alone: $47.3 \%$ vs $36.3 \%$ at 1 year, $28.5 \%$ vs $17.9 \%$ at 2 years, and $20.8 \%$ vs $12.2 \%$ at 3 years. In both trials, ipilimumab was well tolerated and associated with a manageable safety profile. Adverse events were primarily immune-related in nature, consistent with the mechanism of action of ipilimumab. Observations from these and other clinical trials with ipilimumab resulted in product-specific guidelines for managing adverse events to mitigate the impact of immune-related adverse events. ${ }^{9}$

When new drugs are approved, data from use in clinical practice settings may have considerable utility in confirming observations obtained within the controlled setting of phase 3 clinical trials. The present study (CA184-338), a multisite observational chart review, was initiated to gather such data, specifically on first-line treatment with ipilimumab in patients with advanced melanoma in a real-world US treatment setting. Key objectives were to evaluate survival outcomes and safety in patients for whom at least 12 months of follow-up from the start of ipilimumab ther- apy was available, which provides a study population that is generally similar to that treated in the ipilimumab phase 3 trials. Although this report provides results at a median 1 year of follow-up, the study protocol includes yearly updates of survival for this cohort out to 4 years.

\section{Methods}

\section{Study design}

The study was an observational chart review study across 34 US sites and 15 Cytokine Working Group (CWG) and 19 academic/community non-CWG centers, each headed by a medical oncologist physician investigator (PI). Participating sites were instructed to sequentially identify all patients who were treated with ipilimumab and who met study inclusion and exclusion criteria. The PIs and/or their supervised clinical research staff abstracted and transferred existing (retrospective) patient data from medical records onto prequalified, standardized case report forms. Data collection, management, and validation activities were conducted by Medical Data Analytics, a contract research organization (Parsippany, New Jersey).

The study protocol was approved by either the institutional review board (IRB) of the individual participating institution or a centralized review board (New England IRB, Newton, Massachusetts). The need for patient informed consent was determined on a site-by-site basis according to site IRB requirements. If a site required patient consent, then all patients who were still alive and eligible for the study were consented.

\section{Patients}

Eligible patients could have unresectable stage III or metastatic (stage IV, M1a, b, or c) melanoma of all primary types, including cutaneous, noncutaneous, and unknown/unidentifiable. They had to be 18 years or older, at advanced diagnosis, and had to have initiated first-line commercial ipilimumab monotherapy $(3 \mathrm{mg} / \mathrm{kg} \mathrm{q} 3 \mathrm{w} \times 4)$ during April 2011-September 2012. Of note, patients with brain metastases at the time of initiating or continuing ipilimumab were eligible. Key exclusion criteria included any previous systemic treatment for advanced melanoma, current or pending participation in a clinical trial or expanded access program, and concurrent use of therapy for a cancer other than melanoma. Patients who were deceased or alive at the time of data collection were eligible. Patients could have received additional local and/or systemic anticancer therapies, including surgery, radiotherapy, or non-ipilimumab systemic therapy, following the start of ipilimumab treatment, provided these were not initiated at the same time as ipilimumab.

\section{Study variables}

The following study variables were collected and are reported in this analysis: 
- Patient demographics and disease characteristics at diagnosis of advanced melanoma and baseline (ipilimumab initiation);

- Details of ipilimumab dosing, including number of doses administered, doses completed, dose delays, treatment discontinuations, and reasons for delays and discontinuation;

- Concomitant anticancer therapy initiated after the start of and administered during ipilimumab treatment and anticancer therapy provided subsequent to the last dose of ipilimumab initial treatment, including retreatment with ipilimumab;

- Survival-related data, including all-cause death date and date last known to be alive; and

- All adverse events ascribed to disease or treatment (ipilimumab or other anticancer drugs).

Data on adverse events included type of event and whether the event was drug-related, serious, or associated with treatment discontinuation. Adverse events were coded using the Medical Dictionary for Regulatory Activities (MedDRA) version 16.1 and graded according to the Common Terminology Criteria for Adverse Events, version 3.0. ${ }^{10}$

\section{Statistical analysis}

Demographic and disease characteristics at baseline, ipilimumab dosing information, and safety profile data were summarized for all patients. Categorical data were described using counts with percentages and continuous data by medians with ranges.

The primary endpoint for evaluation of effectiveness was $\mathrm{OS}$ in months from the date of ipilimumab initiation. The event of interest was all-cause death; patients who were alive at last follow-up were censored. Median OS and survival rate at 1 year from the date of ipilimumab initiation were estimated for all patients using the Kaplan-Meier method. Corresponding 95\% CI for median OS were calculated using the method of Brookmeyer and Crowley, ${ }^{11}$ and the $95 \% \mathrm{CI}$ for OS rates at 1 year were calculated using the $\log -\log$ transformation. Overall survival was computed in ad hoc, exploratory analyses for specific subgroups, including patients with an Eastern Cooperative Oncology Group Performance Status (ECOG PS) of 0 or 1, without brain metastases at advanced diagnosis, with cutaneous primary site at diagnosis, and by BRAF mutation status. Statistics on ad hoc results were not performed because study was observational. All of the analyses were conducted using SAS version 9 (SAS Institute Inc, Cary, NC).

\section{Results}

A total of 273 patients were included in this study. Data collection was completed during December 2012-January 2013. The median times from initial melanoma diagno- sis to advanced melanoma diagnosis and from advanced melanoma diagnosis to first dose of ipilimumab were 16.6 months (range, 0-312) and 1.2 months (range, 0-72), respectively. The median age was 64 years (range, 26-91) and $64.8 \%$ of patients were men (Table 1 ). At the time of diagnosis of advanced disease, $12.1 \%$ of patients had unresectable stage III disease, $56.0 \%$ had stage IV M1c disease, and $12.1 \%$ had brain metastases. The primary site was cutaneous in $88.3 \%$ of patients. Before receiving ipilimumab, $38.1 \%$ and $42.5 \%$ of patients had ECOG PS 0 or 1, respectively, and $36.6 \%$ had serum lactate dehydrogenase levels greater than the institutional upper limit of normal. BRAF mutation status was known for 231 patients, of whom $50(21.6 \%)$ had a V600 mutation, and $181(78.4 \%)$ were BRAF wild-type.

In all, 78\% of patients received all 4 planned doses of ipilimumab (Supplemental data). The most common reasons for discontinuing dosing were disease progression (10.3\%), drug toxicity (7.0\%), and patient request (3.7\%). Ipilimumab dosing was delayed once in 14 patients (5.1\%), with the most frequent reasons given being fever (1.1\%), hypotension $(0.7 \%)$, and pruritus ( $0.7 \%)$. No patient experienced more than 1 dose delay.

Additional anticancer therapy during ipilimumab treatment was administered to $8.4 \%$ of patients, with radiotherapy being the most frequent type (6.6\%; Supplemental data). Forty-one percent of patients also received supportive care agents, including prednisone (16.1\%), antiemetics $(15.0 \%)$, analgesics (13.9\%), antipruritics (10.6\%), and antimotility agents $(6.6 \%)$. Three patients $(1.1 \%)$ received levothyroxine for thyroiditis, and 1 patient $(<1 \%)$ received infliximab in addition to prednisone for diarrhea/colitis. Subsequent to the last dose of ipilimumab, 126 patients (46.2\%) received additional non-ipilimumab anticancer therapy including surgery (9.9\%), radiotherapy (23.1\%), cytotoxic chemotherapy (17.9\%), targeted therapy (7.7\%), and non-ipilimumab immunotherapy (4.4\%). Eighteen patients $(6.6 \%)$ were retreated with ipilimumab according to standard guidelines and practice for retreatment, which generally require patients to have experienced an objective response or stability lasting at least 3 months and to have had no serious treatment-related toxicity. Overall, 28.9\% of patients received non-ipilimumab systemic therapy subsequent to the last dose of ipilimumab. Twenty-nine percent of patients achieved durable therapeutic benefit and did not receive any further ipilimumab; other patients did not receive further ipilimumab because their performance status worsened (12.8\%) or they died (24.2\%).

At a median follow-up of 12.2 months (interquartile range, 6.6-15.9), 142 (52.0\%) patients had died. Median OS from the start of ipilimumab treatment was 14.5 months (95\% CI, 12.9-18.7), and 1-year survival was 59.2\% (95\% CI, 53.0-64.8) (Table 2, Figure 1). Median 
OS values derived from the ad hoc, exploratory OS analyses for patient subgroups were 21.5 (95\% CI, 16.8-not reached) and 12.8 months (95\% CI, 8.7-17.5) for patients with ECOG PS 0 or 1, respectively; 17.5 months (95\% CI, 13.7-21.5) for patients without brain metastases; and 16.8 months (95\% CI, 13.2-19.8) for patients with a cutaneous primary site of diagnosis. One-year survival rates for BRAF-mutated, wild-type, and untested patients were 71.0\% (95\% CI, 56.0-81.7), 54.9\% (95\% CI, 47.3-61.9), and 63.6\% (95\% CI, 46.9-76.3), respectively.

Adverse events during ipilimumab treatment were reported in 164 patients (60.1\%; Table 3); these were considered drug-related and serious in $53.8 \%$ and $18.7 \%$ of patients, respectively. The most frequently observed adverse events were skin-related (29.3\%), with rash, pruritus, and dermatitis most commonly reported, and gastrointestinalrelated $(27.5 \%)$, the most frequent being diarrhea (Table 4). Drug-related adverse events were grade $3 / 4$ in $15.7 \%$ of patients (13.9 and 1.8, respectively), and there were no toxic deaths. Drug-related adverse events led to discontinuation of treatment in $10.6 \%$ of patients, most often because of colitis (2.9\%), diarrhea (1.8\%), and/or enterocolitis (1.5\%). Of the 48 deaths $(17.6 \%)$ that occurred during treatment, 45 were owing to progressive melanoma, 1 was from nondrug-related congestive heart failure/myocardial infarction, 1 from pulmonary embolism, and 1 from unknown causes.

\section{Discussion}

This multisite observational chart review study is the largest study to date of ipilimumab administered as approved for first-line systemic therapy in patients with advanced melanoma. It is expected that patients treated outside of the controlled setting of a randomized clinical trial may exhibit greater heterogeneity in demographic and disease characteristics than those in a clinical trial cohort. As with any recently approved agent, it was therefore important to assess the effectiveness and safety of ipilimumab observed in routine clinical practice. This real-world study addresses this need and also provides valuable data on the use of ipilimumab at its approved dose and schedule in patients with treatment-naïve advanced melanoma.

Clinical trial experience with ipilimumab $3 \mathrm{mg} / \mathrm{kg}$ monotherapy in treatment-naïve patients with advanced melanoma is limited to 2 phase 2 trials. In the first, 32 patients who were chemotherapy-naive had a median OS of 11.4 months and 1-year survival of $45 \%,{ }^{12}$ and in the second, 40 patients had a median OS of 12.9 months and 1-year survival of $60.9 \% .{ }^{13}$ In the ipilimumab-containing arms of the 2 pivotal phase 3 trials, the median OS range was 10.011.2 months, and the 1 -year survival rate range was $45.6 \%$ $47.3 \% .5,6$ In the present study, the observed median OS of 14.5 months and 1-year survival rate of 59.2\% suggest that survival outcomes with ipilimumab in routine clinical prac-
TABLE 1 Patient demographics and disease characteristics ${ }^{a}$

\begin{tabular}{ll} 
Characteristic & $\begin{array}{l}\text { No. of patients (\%) } \\
(\mathbf{N}=\mathbf{2 7 3})\end{array}$ \\
\hline Male & $177(64.8)$ \\
$\begin{array}{l}\text { Median age: } \\
64 \text { years, range 26-9) }\end{array}$ & - \\
\hline Treated at CWG center & $162(59.3)$ \\
\hline Race & \\
\hline White & $260(95.2)$ \\
Black & $9(3.3)$ \\
Asian & $3(1.1)$ \\
Unknown & $1(<1)$
\end{tabular}

\begin{tabular}{ll} 
Primary site $^{b}$ & \\
\hline Cutaneous & $241(88.3)$ \\
Noncutaneous & $32(11.7)$ \\
Ocular & $12(4.4)$ \\
Mucosal & $5(1.8)$
\end{tabular}

\begin{tabular}{|c|c|}
\hline \multicolumn{2}{|l|}{ Stage ${ }^{c}$} \\
\hline $\begin{array}{l}\text { Stage III (MO) } \\
\text { Stage IV (MI) } \\
\text { Mla } \\
\text { Mlb } \\
\text { Mlc }\end{array}$ & $\begin{array}{l}33(12.1) \\
240(87.9) \\
30(11.0) \\
57(20.9) \\
153(56.0)\end{array}$ \\
\hline \multicolumn{2}{|l|}{ ECOG PS } \\
\hline $\begin{array}{l}0 \\
1 \\
2 \\
3 \\
\text { Unknown }\end{array}$ & $\begin{array}{l}104(38.1) \\
116(42.5) \\
16(5.9) \\
3(1.1) \\
34(12.5)\end{array}$ \\
\hline $\begin{array}{l}\text { Brain metastases } \\
\mathrm{LDH}, \mathrm{n}(\%)^{\mathrm{d}}\end{array}$ & $33(12.1)$ \\
\hline $\begin{array}{l}\text { Elevated } \\
\text { Normal } \\
\text { Not tested }\end{array}$ & $\begin{array}{l}100(36.6) \\
127(46.5) \\
46(16.8)\end{array}$ \\
\hline \multicolumn{2}{|c|}{ BRAF mutation status ${ }^{e}$} \\
\hline $\begin{array}{l}\text { Positive } \\
\text { Negative } \\
\text { Not tested }\end{array}$ & $\begin{array}{l}50(18.3) \\
181(66.3) \\
42(15.4)\end{array}$ \\
\hline
\end{tabular}

CWG, Cytokine Working Group; Eastern Cooperative Oncology Group Performance Status, ECOG PS; LDH, serum lactate dehydrogenase

aAt start of ipilimumab treatment unless otherwise noted. bAt initial diagnosis of melanoma. 'At diagnosis of advanced melanoma. dSite-defined as >upper limit of normal. eAt any melanoma diagnosis.

tice are consistent with those shown in the controlled phase 3 clinical trial setting. Of note, the planned yearly survival updates of this observational cohort will provide real-world data on whether long-term survival is also consistent with the durable long-term survival ( $>3-5$ years) now shown for 20\%-26\% of patients in extended follow-up across the ipi- 
TABLE 2 Efficacy of first-line ipilimumaba

\begin{tabular}{|c|c|}
\hline Efficacy objective & Value \\
\hline \multicolumn{2}{|l|}{ Primary, all patients $(\mathrm{N}=273)^{\mathrm{b}}$} \\
\hline Median OS, mo $(95 \% \mathrm{CI})$ & $\begin{array}{c}14.5 \\
(12.9-18.7)\end{array}$ \\
\hline 1-year survival, \% $(95 \% \mathrm{CI})$ & $\begin{array}{c}59.2 \\
(53.0-64.8)\end{array}$ \\
\hline \multicolumn{2}{|l|}{ Exploratory, for subgroups (ad hoc analyses) } \\
\hline $\begin{array}{l}\text { ECOG PS O }(n=104), \text { median OS, } \\
\text { mo }(95 \% \mathrm{Cl})\end{array}$ & $\begin{array}{l}21.5 \\
(16.8-\mathrm{nr})\end{array}$ \\
\hline $\begin{array}{l}\text { ECOG PS } 1(n=116) \text {, median OS, mo } \\
(95 \% \mathrm{Cl})\end{array}$ & $\begin{array}{c}12.8 \\
(8.7-17.5)\end{array}$ \\
\hline $\begin{array}{l}\text { Without brain metastases }(\mathrm{n}=240) \\
\text { median OS, mo }(95 \% \mathrm{Cl})\end{array}$ & $\begin{array}{c}17.5 \\
(13.7-21.5)\end{array}$ \\
\hline $\begin{array}{l}\text { Cutaneous primary site of diagnosis } \\
\qquad(\mathrm{n}=241) \text {, median OS, mo }(95 \% \mathrm{CI})\end{array}$ & $\begin{array}{c}16.8 \\
(13.2-19.8)\end{array}$ \\
\hline \multicolumn{2}{|l|}{$\begin{array}{l}\text { BRAF mutation status, 1-year survival, } \\
\quad \%(95 \% \mathrm{Cl})^{c}\end{array}$} \\
\hline Positive $(n=50)$ & $\begin{array}{c}71.0 \\
(56.0-81.7)\end{array}$ \\
\hline Wild-type (n = 181) & $\begin{array}{c}54.9 \\
(47.3-61.9)\end{array}$ \\
\hline Not tested $(n=42)$ & $\begin{array}{c}63.6 \\
(46.9-76.3)\end{array}$ \\
\hline
\end{tabular}

Eastern Cooperative Oncology Group Performance Status, ECOG PS; LDH, serum lactate dehydrogenase; OS, overall survival; PS, performance status

aApproved $3 \mathrm{mg} / \mathrm{kg}$ monotherapy $\mathrm{q} 3 \mathrm{w} \times 4$. 'Median follow-up, 12.2 months (interquartile range, 6.6-15.9 months). 'Frequency of BRAF mutations not representative of typical first-line patient population, and may reflect physicians' preference to only treat specific BRAF mutated patients with ipilimumab (eg, patients with less aggressive disease or longer life expectancy).

limumab clinical trials. ${ }^{14}$

As expected, the ad hoc, exploratory OS analyses of subgroups in this study showed higher OS for patients with ECOG PS 0, without brain metastases, and with a cutaneous primary site. BRAF mutation status did not seem to affect survival. These results must be interpreted with caution, however, because this was an observational study with inherent limitations. Indeed, it should be noted that the frequency of BRAF mutations reported here (21.6\%) is lower than the $40 \%-60 \%$ expected in a population of patients with primarily cutaneous melanomas. ${ }^{7}$ This finding may reflect the practice of preferentially treating patients with BRAF-mutated tumors with BRAF inhibitors, particularly patients with unfavorable clinical characteristics. In addition, during the time period covered in this study, clinical trials of BRAF inhibitors in first-line therapy for melanoma were ongoing and probably captured some patients who might otherwise have been treated first-line with ipilimumab. It remains uncertain whether patients with BRAF mutated tumors should receive MAPK inhibitors or ipilimumab as first-line systemic therapy while pro-
TABLE 3 Overall safety with first-line ipilimumaba

\begin{tabular}{|c|c|c|c|}
\hline \multirow[t]{2}{*}{ Adverse events } & \multicolumn{3}{|c|}{$\begin{array}{l}\text { Patients with worst grade, n (\%) } \\
\qquad(N=273)\end{array}$} \\
\hline & Any grade & Grade 3 & Grade 4 \\
\hline Total & $164(60.1)$ & 45 (16.5) & $8(2.9)$ \\
\hline Drug-related & $147(53.8)$ & 38 (13.9) & $5(1.8)$ \\
\hline Serious & $51(18.7)$ & $33(12.1)$ & $7(2.6)$ \\
\hline Drug-related & $38(13.9)$ & $26(9.5)$ & $5(1.8)$ \\
\hline $\begin{array}{l}\text { Leading to } \\
\text { discontinuation }\end{array}$ & $31(11.4)$ & $18(6.6)$ & $4(1.5)$ \\
\hline Drug-related & $29(10.6)$ & $18(6.6)$ & $3(1.1)$ \\
\hline \multicolumn{4}{|c|}{$\begin{array}{l}\text { approved } 3 \mathrm{mg} / \mathrm{kg} \text { monotherapy } \mathrm{q} 3 \mathrm{w} \times 4 \text {; restricted to adverse events reported between } \\
\text { the first ipilimumab dose and earliest of } 70 \text { days after the last initial dose or, if ipilimumab } \\
\text { was given again, the day before the start of retreatment. }{ }^{b} \text { Common Terminology Criteria, } \\
\text { Version } 3.0 \text {; there were no Grade } 5 \text { adverse events. CPatients may have experienced more } \\
\text { than one adverse event. }\end{array}$} \\
\hline
\end{tabular}

spective studies are ongoing to inform clinical practice. ${ }^{15}$ Retrospective data suggest that response to a BRAF inhibitor is similar pre- and post-ipilimumab, but response to ipilimumab may be improved before a BRAF inhibitor compared with after a BRAF inhibitor. ${ }^{16}$ On the basis of that evidence, the use of ipilimumab before a BRAF inhibitor has been recommended, particularly in an initial setting of asymptomatic or low-volume disease. In the future, however, a potential strategy may be to start with targeted therapy and then to switch to immunotherapy at the time of best response (before failure occurs). ${ }^{16,17}$

Patients with melanoma in the US are treated in both academic and community centers, but patients with advanced melanoma in the European Union and many other regions are treated primarily in centralized academic centers. Although the present study was limited to centers in the US, they were primarily specialized centers (CWG or CWG-affiliated). Thus, patients in this cohort were treated by academic clinicians who had clinical trial experience with ipilimumab treatment, selection of appropriate patients, and managing immune-related adverse events, and who were supported by similarly experienced and dedicated clinical and research teams. This arguably makes the study results generalizable to the average treatment-naive patient with advanced melanoma who might be treated with first-line ipilimumab therapy in centralized academic centers in the European Union and other regions. In contrast to the primarily academic setting of our study, the community setting (McKesson Specialty Health/US Oncology Network) was exclusively the source of patients for another US multisite, retrospective, observational cohort study that was conducted with the same objectives during the same timeframe. ${ }^{18}$ In this second study, median OS was 11.5 months (95\% CI, 8.9-16.6) and 1-year survival was 


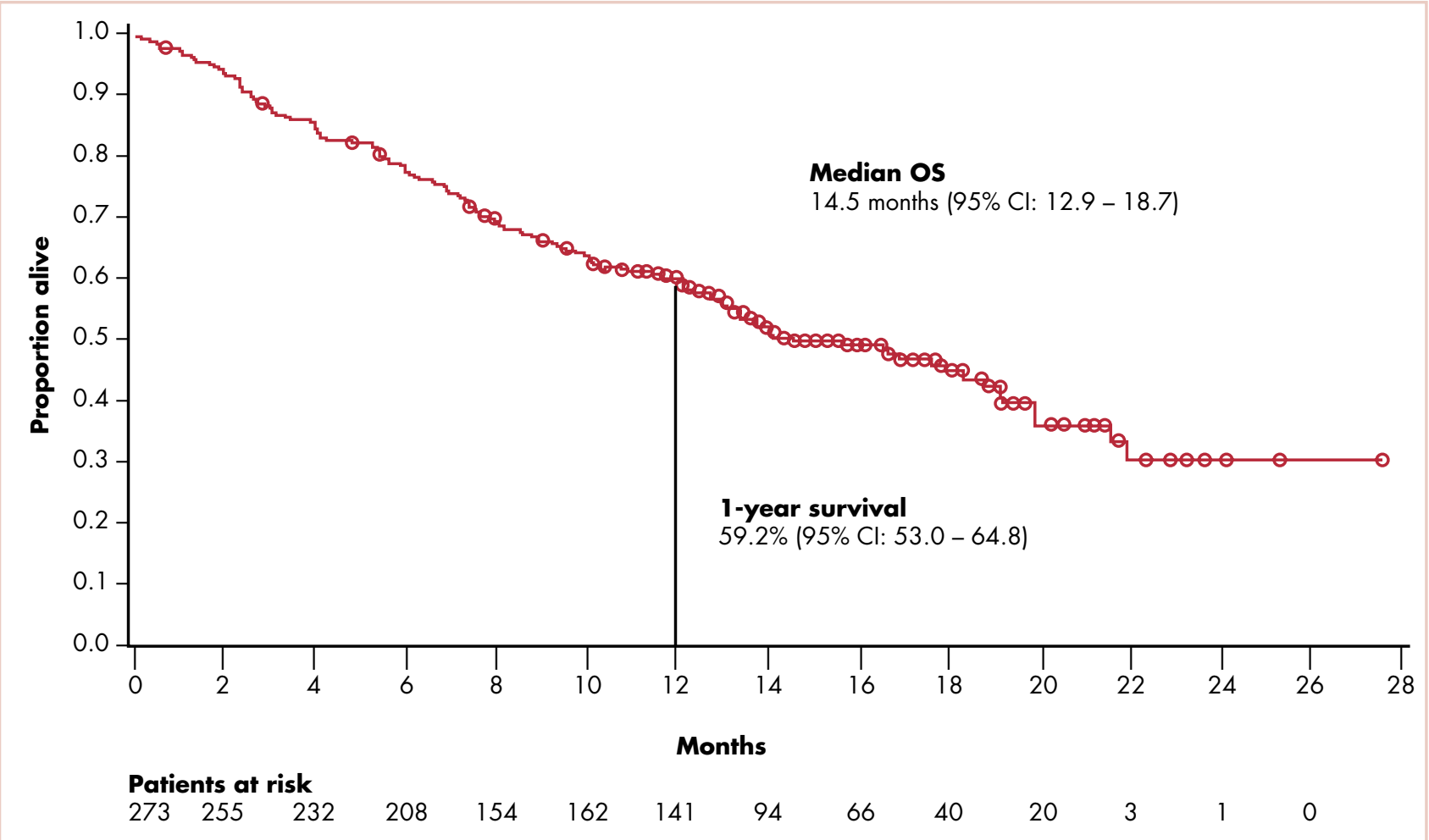

FIGURE 1 Overall survival from start of first-line ipilimumab. $\mathrm{N}=273$; median follow-up, 12.2 months (interquartile range: 6.6 , 15.9 months). Circles indicate censored observations.

46.7\% (95\% CI, 38.1-54.9) for the 157-patient cohort with treatment-naïve advanced melanoma who received ipilimumab monotherapy. Fewer patients in this community setting cohort received all 4 cycles of the first-line ipilimumab $(53.8 \%$ vs $77.7 \%$ in the present study) and this population also had a higher proportion of patients with brain metastases (34.4\% vs $12.1 \%$ in the present study); both of those differences could have contributed to the lower survival observed in the second study. Despite the differences, the results of these 2 observational studies are independently consistent with those from the ipilimumab clinical trials, which strongly suggest that improved survival can be achieved with ipilimumab in the real-world treatment settings of both academic and community practice.

Ipilimumab seemed to be well tolerated in this treatment-naïve cohort, with nearly $80 \%$ of patients able to receive all 4 of the prescribed doses of ipilimumab. This therapy was also associated with a safety profile similar to that observed in clinical trials of ipilimumab at this dose and schedule. In those trials, the most frequent adverse events occurred in the skin and gastrointestinal systems. Grade 3/4 drug-related adverse events were reported in $15.7 \%$ (13.9\% and $1.18 \%$, respectively) of patients in the present study, and in 17.4\%-22.9\% (16.3-19.1 and 1.1-3.8) of previously treated patients receiving ipilimumab $3 \mathrm{mg} /$ $\mathrm{kg}$ in the first pivotal phase 3 study. ${ }^{6}$ Of note is that there were no adverse event-related deaths or gastrointestinal perforations reported in this study, which possibly reflects careful patient selection and vigilant use of the ipilimumab adverse event management guidelines, which call for rigorous monitoring and prompt intervention with immunosuppressive therapy. ${ }^{9}$ This intervention, starting with glucocorticosteroids and sometimes requiring the addition of adjunctive immunosuppressants such as infliximab (in patients whose immune-related adverse events are not well controlled by steroids or to spare patients the risks inherent with long-term and/or high-dose glucocorticosteroid therapy), was shown to prevent the worsening of immunerelated events and to treat them effectively in nearly all cases in the phase 3 trials. ${ }^{5,6}$ The results suggest that the use of these adverse event-management guidelines in a realworld setting yields a manageable safety profile similar to that observed in the ipilimumab clinical trials.

This study is limited by its observational nature and the heterogeneity of patients available for inclusion in the report. On the one hand, the inclusion here of patients typically excluded from most ipilimumab clinical studies (eg, those with brain metastases) and others who would also be expected to have a poorer prognosis (eg, with ocular or mucosal melanoma) could unfavorably affect the appar- 


\begin{tabular}{|c|c|c|c|c|c|c|}
\hline \multirow{2}{*}{ Adverse event } & \multicolumn{6}{|c|}{ Patients with worst grade, $n(\%)^{b}(N=273)$} \\
\hline & Grade 1 & Grade 2 & Grade 3 & Grade 4 & Unknown & Any Grade \\
\hline Any ${ }^{c}$ & $42(15.4)$ & $67(24.5)$ & $45(16.5)$ & $8(2.9)$ & $2(0.7)$ & $164(60.1)$ \\
\hline Skin & $49(17.9)$ & $24(8.8)$ & $5(1.8)$ & 0 & $2(0.7)$ & $80(29.3)$ \\
\hline Rash & $27(9.9)$ & $11(4.0)$ & $2(0.7)$ & 0 & 0 & 40 (14.7) \\
\hline Pruritus & $22(8.1)$ & $12(4.4)$ & $1(0.4)$ & 0 & $1(0.4)$ & $36(13.2)$ \\
\hline Dermatitis & $17(6.2)$ & $7(2.6)$ & $2(0.7)$ & 0 & $1(0.4)$ & $27(9.9)$ \\
\hline Gastrointestinal & $25(9.2)$ & $26(9.5)$ & $21(7.7)$ & $2(0.7)$ & 1 & $75(27.5)$ \\
\hline Diarrhea & 20 (7.3) & $16(5.9)$ & $4(1.5)$ & 0 & 0 & 40 (14.7) \\
\hline Colitis & $2(0.7)$ & $6(2.2)$ & $10(3.7)$ & $1(0.4)$ & 0 & $19(7.0)$ \\
\hline Nausea & $8(2.9)$ & $5(1.8)$ & $1(0.4)$ & 0 & 0 & $14(5.1)$ \\
\hline Fatigue & $16(5.9)$ & $31(11.4)$ & $8(2.9)$ & 0 & 0 & $55(20.1)$ \\
\hline Nervous system ${ }^{d}$ & $9(3.3)$ & $10(3.7)$ & $5(1.8)$ & $2(0.7)$ & $1(0.4)$ & $27(9.9)$ \\
\hline Endocrine $^{e}$ & $2(0.7)$ & $8(2.9)$ & $6(2.2)$ & $1(0.4)$ & 0 & $17(6.2)$ \\
\hline
\end{tabular}

${ }^{a}$ Approved $3 \mathrm{mg} / \mathrm{kg}$ monotherapy $\mathrm{q} 3 \mathrm{w} \times 4$; restricted to adverse events reported between the first ipilimumab dose and earliest of 70 days after the last initial dose or, if ipilimumab was given again, the day before the start of retreatment. bCommon Terminology Criteria, Version 3.0; there were no Grade 5 adverse events. cPatients may have experienced more than one adverse event. dMost common adverse event of any grade was peripheral neuropathy in 12 (4.4\%) patients. Headache was the most common Grade 3 adverse event, occurring in $3(1.1 \%)$ patients. eMost common Grade 3 or 4 adverse events were adrenal insufficiency and hypophysitis in 3 (1.1\%) patients each.

ent activity and toxicity experience with ipilimumab. On the other hand, the exclusion of patients receiving previous therapy for advanced disease could favorably bias the data. Further limitations are imposed by the incomplete nature of some data, loss to follow-up, and possible overor under-reporting of selected outcomes, especially adverse events. For the OS analysis, the censoring of patients not known to have died by the time of the data lock reduces the reliability of the OS results with increasing time; its impact is expected to decline with longer follow-up in this cohort. Finally, the OS results are confounded by the unknown contribution of additional anticancer therapy provided during and subsequent to initial ipilimumab therapy, including ipilimumab retreatment.

In conclusion, this report expands our knowledge of the clinical profile of ipilimumab in patients with advanced melanoma who received the drug as first-line therapy in a real-world clinical practice setting. To our knowledge, it is the largest study of first-line ipilimumab for patients with advanced melanoma treated outside of a clinical trial to date, and it included patients across all primary subtypes of melanoma, including noncutaneous subtypes and those with brain metastases, for whom clinical trial data are limited. ${ }^{19,20}$ The observed median OS of 14.5 months and 1-year survival rate of $59.2 \%$ provide evidence that the improved survival outcomes associated with ipilimumab treatment in the pivotal clinical trials are achievable in a real-world clinical setting. Although these results are subject to the limitations of data obtained from observational studies, they support the effectiveness and safety of ipilimumab used at the indicated $3 \mathrm{mg} / \mathrm{kg}$ dose and schedule as first-line therapy for patients with advanced melanoma.

\begin{abstract}
Acknowledgments
This study was presented in part at the 2013 European Cancer Congress, October 2013, and the 2013 Society for Melanoma Research International Congress, November 2013. The authors take full responsibility for the content of this publication and confirm that it reflects their viewpoint and medical expertise. Data collection, research operations, and analytics support were provided by Schiffon L Wong, Marianne Messina, and John R Penrod of Bristol-Myers Squibb; by and Pete Wolthoff, Cynthia Macahilig, and Scott Merrill of Medical Data Analytics. Professional medical writing assistance was provided by Jennifer Wietzke and Ward A. Pedersen at StemScientific, an Ashfield Company, funded by Bristol-Myers Squibb.
\end{abstract}

\section{References}

1. American Cancer Society, Cancer Facts \& Figures 2015. http:// www.cancer.org/acs/groups/content/@editorial/documents/document/acspc-044552.pdf. Accessed March 19, 2015.

2. Forsea AM, Del Marmol V, de Vries E, Bailey EE, Geller AC Bailey. Melanoma incidence and mortality in Europe: new estimates, persistent disparities. Br J Dermatol. 2012;167:1124-1150.

3. Korn EL, Liu PY, Lee SJ, et al. Meta-analysis of phase II cooperative group trials in metastatic stage IV melanoma to determine progression-free and overall survival benchmarks for future phase II trials. J Clin Oncol. 2008;26:527-534.

4. Garbe C, Eigentler TK, Keilholz U, Hauschild A, Kirkwood JM. Systematic review of medical treatment in melanoma: current status and future prospects. Oncologist. 2011;16:5-24.

5. Hodi FS, O'Day SJ, McDermott DF, et al. Improved survival with ipilimumab in patients with metastatic melanoma. $\mathrm{N}$ Engl J Med. 2010;363:711-723.

6. Robert C, Thomas L, Bondarenko I, et al. Ipilimumab plus dacarbazine for previously untreated metastatic melanoma. N Engl J Med. 2011;364:2517-2526.

7. Chapman PB, Hauschild A, Robert C, et al. Improved survival 


\section{Original Report}

with vemurafenib in melanoma with BRAF V600E mutation. N Engl J Med. 2011;364:2507-2516.

8. Luke JJ, Hodi FS. Ipilimumab, vemurafenib, dabrafenib, and trametinib: synergistic competitors in the clinical management of BRAF mutant malignant melanoma. Oncologist. 2013;18:717-725.

9. Weber JS, Kahler KC, Hauschild A. Management of immune-related adverse events and kinetics of response with ipilimumab. J Clin Oncol. 2012;30:2691-2697.

10. Cancer Therapy Evaluation Program. Common Terminology Criteria for Adverse Events (CTCAE). Version 3.0, DCTD, NCI, NIH, DHHS. August 9, 2006. http://ctep.cancer.gov/protocolDevelopment/electronic_applications/docs/ctcaev3.pdf. Accessed March 1, 2013.

11. Brookmeyer R, Crowley J. A confidence interval for the median survival time. Biometrics. 1982;38:29-41.

12. Hersh EM, O'Day SJ, Powderly J, et al. A phase II multicenter study of ipilimumab with or without dacarbazine in chemotherapy-naive patients with advanced melanoma. Invest New Drugs. 2011;29:489498.

13. Hamid $\mathrm{O}$, Schmidt $\mathrm{H}$, Nissan A, et al. A prospective phase II trial exploring the association between tumor microenvironment biomarkers and clinical activity of ipilimumab in advanced melanoma. J Trans Med. 2011;9:204.
14. Schadendorf D, Hodi FS, Roberts C, et al. Pooled analysis of longterm survival data from phase II and phase III trials of ipilimumab in unresectable or metastatic melanoma. J Clin Oncol. 2014: submitted March 2014.

15. Jang S, Atkins MB. Which drug, and when, for patients with BRAFmutant melanoma? Lancet Oncol. 2013;14:e60-e69.

16. Ackerman A, Klein O, McDermott DF, et al. Outcomes of patients with metastatic melanoma treated with immunotherapy prior to or after BRAF inhibitors. Cancer. 2014; 120:1695-1701.

17. Ascierto PA, Margolin K. Ipilimumab before BRAF inhibitor treatment may be more beneficial than vice versa for the majority of patients with advanced melanoma. Cancer. 2014;120:1617-1619.

18. Patt D, Rembert D, Bhor M, Bhowmik D, Rao S. A real-world observational study of patients with advanced melanoma receiving first-line Ipilimumab in a community practice setting. J Cancer Ther 2014: submitted March 2014

19. Margolin K, Ernstoff MS, Hamid O, et al. Ipilimumab in patients with melanoma and brain metastases: an open-label, phase 2 trial. Lancet Oncol. 2012;13:459-465.

20. Di Giacomo AM, Ascierto PA, Pilla L, et al. Ipilimumab and fotemustine in patients with advanced melanoma (NIBIT M1): an openlabel, single-arm phase 2 trial. Lancet Oncol. 2012;13:879-886. 